AIAA 95-0478

Airfoil Design and Optimization

by the One-Shot Method

G. Kuruvila

ViGYAN, Inc., Hampton, VA

Shlomo Ta'asan

Carnegie Mellon University, Pittsburgh, PA

M.D. Salas

NASA Langley Research Center, Hampton, VA

\author{
33rd Aerospace Sciences \\ Meeting and Exhibit \\ January 9-12, 1995 / Reno, NV
}





\title{
Airfoil Design and Optimization by the One-Shot Method
}

\author{
G. Kuruvila* \\ ViGYAN, Inc. \\ Hampton, VA 23666-1325 \\ Shlomo Ta'asan ${ }^{\dagger}$ \\ Carnegie Mellon University \\ Pittsburgh, PA 15213-3890 \\ M. D. Salas \\ NASA Langley Research Center \\ Hampton, VA 23681-0001
}

\begin{abstract}
An efficient numerical approach for the design of optimal aerodynamic shapes is presented in this paper. The objective of any optimization problem is to find the optimum of a cost function subject to a certain state equation (governing equation of the flow field) and certain side constraints. As in classical optimal control methods, the present approach introduces a costate variable (Lagrange multiplier) to evaluate the gradient of the cost function. High efficiency in reaching the optimum solution is achieved by using a multigrid technique and updating the shape in a hierarchical manner such that smooth (low-frequency) changes are done separately from high-frequency changes. Thus, the design variables are changed on a grid where their changes produce nonsmooth (high-frequency) perturbations that can be damped efficiently by the multigrid. The cost of solving the optimization problem is approximately two to three times the cost of the equivalent analysis problem.
\end{abstract}

\section{Nomenclature}

F cost function

$f_{k} \quad k$ th shape function

$M_{\infty} \quad$ free-stream Mach number

$n \quad$ unit normal

$\boldsymbol{t}$ unit tangent

$\boldsymbol{U}_{\infty} \quad$ free-stream velocity

$y^{\mathrm{U}, \mathrm{L}} \quad y$-coordinate of the upper and lower surface of the airfoil

$\boldsymbol{\alpha} \quad$ amplitude of shape functions (design variables)

$\tilde{\boldsymbol{\alpha}} \quad$ direction of change of $\boldsymbol{\alpha}$

$\alpha_{k}^{\mathrm{U}, \mathrm{L}} \quad$ components of $\boldsymbol{\alpha}$ (upper and lower surface amplitudes of the $k$ th shape function)

$\Gamma \quad$ circulation

$\gamma \quad$ ratio of specific heats

$\varepsilon \quad$ magnitude of change of $\boldsymbol{\alpha}$

$\zeta \quad$ angle of attack

$\Theta \quad \theta$ corrected for Mach number

\footnotetext{
${ }^{*}$ Research Engineer; Senior Member AIAA.

$\dagger$ Associate Professor.

$¥$ Computational AeroSciences Team Leader.

Copyright (C) 1994 by the American Institute of Aeronautics and Astronautics, Inc. No copyright is asserted in the United States under Title 17, U.S. Code. The U.S. Government has a royaltyfree license to exercise all rights under the copyright claimed herein for Governmental purposes. All other rights are reserved by the copyright owner.
}

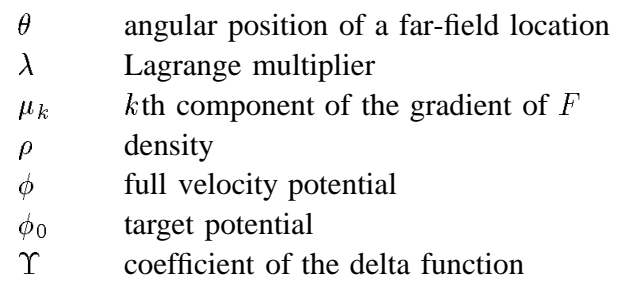

\section{Introduction}

The aerodynamic designer seeks an airplane shape that achieves the best aerodynamic performance while taking into account the trade-offs with other disciplines, namely, structures, propulsion, stability, and control. Although significant progress has been made in developing computational methods for fluid flow analysis, the methods for the design and optimization of aircraft configuration are still in their infancy. Among the many techniques that have been developed, the inverse design method ${ }^{1-6}$ is perhaps the most widely used. This method is severely restrictive because its depends on the experience and knowledge of the designer to establish desirable velocity or pressure distributions. In addition, the method does not lend itself to the imposition of constraints. The next most popular approach is what we refer to as the "loosely coupled optimization (LCO)." In this approach, the analysis problem is solved many times to find the gradient of the cost function with finite differences. This gradient is used by a "black box" optimizer to find the best change to the design variables. The computational cost of this brute-force 
method is usually exorbitant. Examples of this approach are found in Refs. 7-10. The LCO method can be improved by analytically evaluating the sensitivity derivatives that are needed to update the design variables. ${ }^{11}$ Usually, this requires the inversion of an extremely large matrix. For threedimensional problems, the size of this matrix can render the method impractical with current computer technology. In the past several years, the adjoint variable method has gained popularity in the area of aerodynamic design. This method, which we refer to as "tightly coupled optimization (TCO)," requires the solution of an adjoint problem, in addition to the analysis problem, to evaluate the gradient of the cost function. The complexity of the adjoint problem is equivalent to that of the analysis problem. However, the adjoint problem, along with the analysis problem, must be solved many times to reach convergence. This approach has been discussed in Refs. 12 and 13. Although the efficiency of TCO is much higher than that of LCO, this procedure can also become prohibitively expensive for practical aerodynamic design and optimization problems.

The One-Shot method ${ }^{14,15}$ overcomes the unacceptable cost of the existing design and optimization procedures. It brings the cost of design and optimization to the same order as that of a single analysis. High performance is achieved both by exploiting the property of the partial differential equations (associated with the scales (frequency) of the errors) which govern the physics of the flow and by the efficient damping of high-frequency error components with multigrid. Consider the subsonic flow over an airfoil profile. The change in the shape of the profile of a given wavelength produces changes of the same wavelength in the solution. These changes penetrate into the flow field only up to a distance that is proportional to the wavelength of the perturbation. Thus, while the high-frequency changes in the shape of the airfoil produce changes in the solution that are of high frequency and remain local to the neighborhood of the airfoil, the smooth (low-frequency) changes in the shape produce smooth changes to the solution and are global in nature. Typically, any relaxation scheme quickly damps the high-frequency components of the error on a grid. Multigrid efficiently damps the whole spectrum of error components by relaxing the governing equations on a sequence of grids of varying resolution.

Therefore, the basic idea of the One-Shot method is to change the shape of the airfoil profile in a hierarchical manner such that smooth changes are made separately from highfrequency changes. Because each of these changes involves a different scale, the governing equation of the flow field can be solved efficiently on grids of appropriate resolution. Thus, the flow field due to smooth changes in the shape of the airfoil is solved on coarse grids, and the flow field due to increasingly high-frequency shape changes is solved on increasingly fine grids. This breaks the optimization procedure into a sequence of suboptimization problems, each of a given scale; therefore, the problem is well conditioned. The resulting optimization procedure is very efficient because the work on a particular scale is done on the appropriate grid. (Ill conditioning results from working on many scales simultaneously.) The OneShot method is implemented within a full approximation scheme (FAS) full multigrid (FMG) algorithm. The solution process starts on the coarsest grid, where only the smooth component of the shape function is updated. This solution is interpolated to the next finest grid, where it serves as an initial approximation of the solution on that grid. This process is continued until the finest grid is reached. Thus, smooth (low-frequency) shapes are updated on coarse grids; high-frequency shapes are updated on finer grids. The fine- to coarse-grid transfers are designed such that the optimization problem at each grid level is driven by the fine-grid residual. The resulting algorithm has an estimated overall cost that ranges from two to three times the cost of the analysis problem.

The successful application of the One-Shot method to the aerodynamic shape design problem was first reported in Ref. 15. The capability of the method was demonstrated by using the small-disturbance potential equation as the governing equation of the flow field. However, in that study, the issue of updating the grid was avoided. In the present study, the full potential equation is used as the governing equation; hence, the grid must be updated as the shape changes. In this work, the adjoint equation and the corresponding gradient of the cost function are derived. The solution procedure and some typical results are also presented.

\section{Design of Optimal Airfoil Shapes}

The design of optimal airfoil shapes is a constrained minimization problem. The objective is to find the optimal shape of the airfoil that will minimize a cost function $F$ subject to the state equation of the flow field and the side constraints.

\section{The State Equations}

The analysis problem, defined by the state equation, consists of finding the flow over a specified shape for a given free-stream Mach number and angle of attack. In order to focus on the optimization procedure, the flow model considered is the subsonic potential flow over an airfoil profile.

Consider the steady irrotational flow past a twodimensional airfoil. ${ }^{16,17}$ The governing equation of the flow field, known as the full potential equation, is

$$
\operatorname{div}(\rho \nabla \phi)=0
$$

The boundary condition on the airfoil is

$$
\nabla \phi \cdot \boldsymbol{n}=0
$$

At infinity, the boundary condition is

$$
\nabla \phi=\boldsymbol{U}_{\infty}
$$




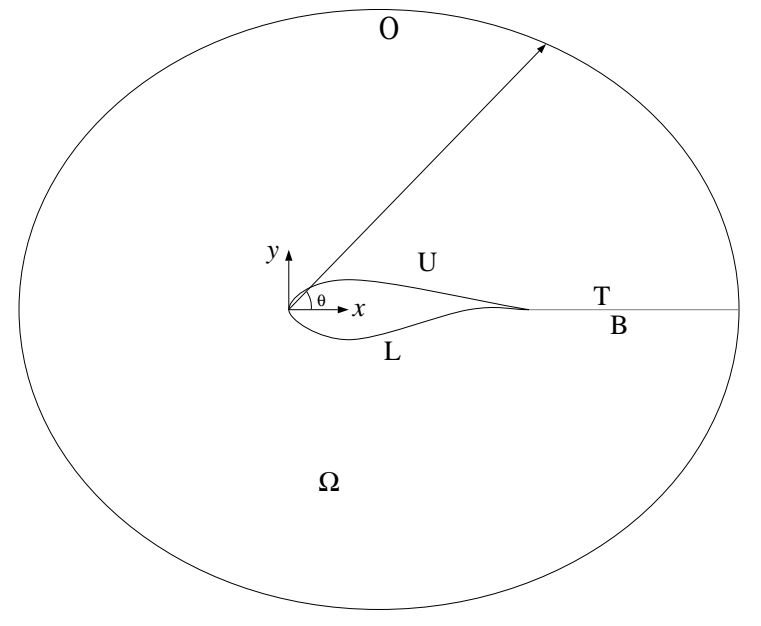

Fig. 1. Computational domain.

For the Kutta condition, the circulation $\Gamma$ around the airfoil is such that

the velocity at the trailing edge is finite and continuous

In these equations, $\phi=\phi(x, y)$ is the full velocity potential, $\rho=\rho(\phi)$ is the density, $\boldsymbol{n}$ is the unit normal, and $\boldsymbol{U}_{\infty}$ is the free-stream velocity. The density $\rho$ is given by

$$
\rho=\left[1-\frac{\gamma-1}{2} M_{\infty}^{2}\left(|\nabla \phi|^{2}-1\right)\right]^{\frac{1}{\gamma-1}}
$$

where $M_{\infty}$ is the free-stream Mach number and $\gamma$ is the ratio of specific heats. If $\zeta$ is the angle of attack of the airfoil, then the free-stream velocity is given by

$$
\boldsymbol{U}_{\infty}=U_{\infty}[\cos (\zeta) \boldsymbol{i}+\sin (\zeta) \boldsymbol{j}]
$$

where $i$ and $j$ are the unit vectors in the $x$ and $y$ directions, respectively.

\section{The Computational Domain}

The computational domain is shown in Fig. 1. The interior of the flow field is denoted by $\Omega$; the upper and lower surfaces of the airfoil are denoted by $U$ and $L$, respectively. The far-field boundary, located at a finite distance from the airfoil (30 to 50 airfoil chord lengths) is denoted by $\mathrm{O}$. To impose the Kutta condition around the airfoil, an artificial boundary or cut that begins at the airfoil and extends to the far field is introduced. A jump in potential that is equal to $\Gamma$ is allowed across the cut. For convenience, this cut is chosen to emanate from the trailing edge of the airfoil. The top and bottom sides of the cut are denoted by $\mathrm{T}$ and $\mathrm{B}$, respectively. The jump across the cut can be written as

$$
\phi^{\mathrm{T}}-\phi^{\mathrm{B}}=\Gamma
$$

The value of the $\Gamma$ is determined by requiring that the velocity perpendicular to the trailing edge bisector be equal to 0 at the trailing edge. A good approximation for $\Gamma$ is given by

$$
\Gamma=\phi_{t . e .}^{\mathrm{T}}-\phi_{t . e .}^{\mathrm{B}}
$$

where t.e. refers to the trailing edge of the airfoil. To satisfy mass conservation across the cut, derivatives of the potential normal to the cut are required to be continuous.

At the far-field boundary, the circulation modifies the velocity as follows:

$$
\nabla \phi \cdot \boldsymbol{n}=\boldsymbol{U}_{\infty} \cdot \boldsymbol{n}+\frac{\Gamma}{2 \pi} \nabla \Theta \cdot \boldsymbol{n}
$$

where

$$
\Theta=2 \pi-\tan ^{-1}\left(\sqrt{1-M_{\infty}^{2}} \tan \theta\right)
$$

and $\theta$ is the angular position of a far-field point. For convenience, $\boldsymbol{n}$ is the unit normal on the boundary. The far-field boundary condition given by (9) is consistent with the infinity condition stated by (3).

\section{The Design Variables}

The airfoil is represented as follows:

$$
\begin{aligned}
& y^{\mathrm{U}}=\sum_{k=1}^{K} \alpha_{k}^{\mathrm{U}} f_{k}(x) \\
& y^{\mathrm{L}}=\sum_{k=1}^{K} \alpha_{k}^{\mathrm{L}} f_{k}(x) \\
& (0 \leq x \leq 1)
\end{aligned}
$$

where $\alpha_{k}^{\mathrm{U}}$ and $\alpha_{k}^{\mathrm{L}}$ are the amplitudes of the shape functions $f_{k}$ on the upper and lower surfaces of the airfoil, respectively. The design variables $\alpha_{k}$ must be determined to obtain the optimal shape of the airfoil. Let $\boldsymbol{\alpha}$ denote a vector whose elements are the design variables. That is,

$$
\boldsymbol{\alpha}=\left[\alpha_{1}^{\mathrm{U}}, \alpha_{2}^{\mathrm{U}}, \ldots, \alpha_{K}^{\mathrm{U}}, \alpha_{1}^{\mathrm{L}}, \alpha_{2}^{\mathrm{L}}, \ldots, \alpha_{K}^{\mathrm{L}}\right]^{T}
$$

The functionality of the shape functions will be presented later.

\section{The Optimization Problem}

The model problem chosen is the design of an airfoil shape that can match a given target potential. Given a target potential distribution $\phi_{0}$ around an airfoil, the objective is to find $\boldsymbol{\alpha}$ that will minimize

$$
F[\boldsymbol{\alpha}, \phi(\boldsymbol{\alpha})] \equiv \int_{\mathrm{U}+\mathrm{L}}\left(\phi-\phi_{0}\right)^{2} d \sigma
$$

subject to the state equations, where $d \sigma$ (which is an element of the airfoil) can be written as

$$
d \sigma^{2}=d x^{2}+d y^{2}
$$

Note that the choice of this particular cost function does not make it an inverse-design problem. Unlike inverse-design problems, the minimization is done over a finite number of 
design variables. This approach also can be used, for example, to find the optimal shape of an airfoil that has the minimum $D / L$ (drag/lift) subject to geometric and aerodynamic constraints.

To make the presentation of the derivation of the adjoint equations simple and easy to understand, the flow is assumed to be incompressible (i.e., $M_{\infty}=0$ ); therefore, $\rho=1$. In this case, the full potential equation reduces to the Laplace equation. Also, no side constraints are considered in this derivation. Therefore, the specific optimization problem considered here is

$$
\min _{\alpha, \phi} \int_{\mathrm{U}+\mathrm{L}}\left(\phi-\phi_{0}\right)^{2} d \sigma
$$

subject to

$$
\begin{aligned}
\operatorname{div}(\nabla \phi) & =0 & & \text { in } \Omega \\
\nabla \phi \cdot \boldsymbol{n} & =0 & & \text { on the airfoil } \\
\nabla \phi \cdot \boldsymbol{n} & =\boldsymbol{U}_{\infty} \cdot \boldsymbol{n}+\frac{\Gamma}{2 \pi} \nabla \Theta \cdot \boldsymbol{n} & & \text { in the far field } \\
\phi^{\mathrm{T}}-\phi^{\mathrm{B}} & =\Gamma & & \text { along the cut }
\end{aligned}
$$

where $\Gamma$ is given by (8).

\section{The Minimization Process}

At some initial $\boldsymbol{\alpha}$, any minimization process seeks to find a descent direction $\tilde{\boldsymbol{\alpha}}$ and a step size $\varepsilon$ in which to change $\boldsymbol{\alpha}$ such that

$$
F(\boldsymbol{\alpha}+\varepsilon \tilde{\boldsymbol{\alpha}}, \phi+\varepsilon \tilde{\phi}) \leq F(\boldsymbol{\alpha}, \phi)
$$

where $\varepsilon \tilde{\phi}$ is the corresponding change in $\phi$ that satisfies the state equations. This process is repeated several times until a minimum is reached.

The Descent Direction. A descent direction $\tilde{\boldsymbol{\alpha}}$ can be determined as follows. The Taylor series expansion of $F$ about $\boldsymbol{\alpha}$ and $\phi$ can be written as

$$
F(\boldsymbol{\alpha}+\varepsilon \tilde{\boldsymbol{\alpha}}, \phi+\varepsilon \tilde{\phi})=F(\boldsymbol{\alpha}, \phi)+\varepsilon \tilde{\boldsymbol{\alpha}}^{T} \nabla_{\boldsymbol{\alpha}} F(\boldsymbol{\alpha}, \phi)+O\left(\varepsilon^{2}\right)
$$

where

$$
\nabla_{\boldsymbol{\alpha}} F \equiv \frac{\partial F}{\partial \boldsymbol{\alpha}}+\left(\frac{d \phi}{d \boldsymbol{\alpha}}\right)^{T} \frac{\partial F}{\partial \phi}
$$

Equation (18) clearly shows that if

$$
\tilde{\boldsymbol{\alpha}}=-\frac{\nabla_{\boldsymbol{\alpha}} F(\boldsymbol{\alpha}, \phi)}{\left|\nabla_{\boldsymbol{\alpha}} F(\boldsymbol{\alpha}, \phi)\right|}
$$

then (17) is satisfied. Equality occurs in (17) at the minimum when $\nabla_{\boldsymbol{\alpha}} F\left(\boldsymbol{\alpha}^{*}, \phi^{*}\right)=0$, where $\boldsymbol{\alpha}^{*}$ is the optimum value of the design variables and $\phi^{*}$ is the corresponding value of the state variables that satisfies the state equations. Therefore, to obtain the descent direction, the gradient of $F$ must be evaluated.
The Step Size. The step size $\varepsilon$ is determined by a line search. The objective of the line search is to find $\varepsilon$ such that $\left\|\nabla_{\boldsymbol{\alpha}} F(\boldsymbol{\alpha}+\varepsilon \tilde{\boldsymbol{\alpha}}, \phi+\varepsilon \tilde{\phi})\right\|^{2}$ is a minimum. That is,

$$
\frac{\partial\left\|\nabla_{\boldsymbol{\alpha}} F(\boldsymbol{\alpha}+\varepsilon \tilde{\boldsymbol{\alpha}}, \phi+\varepsilon \tilde{\phi})\right\|^{2}}{\partial \varepsilon}=0
$$

By expanding in Taylor's series, taking the derivative with respect to $\varepsilon$, and neglecting the $O\left(\varepsilon^{2}\right)$ terms, the step size becomes

$$
\varepsilon=-\frac{\left[\nabla_{\boldsymbol{\alpha}} F(\boldsymbol{\alpha}, \phi)\right]^{T} \nabla_{\boldsymbol{\alpha}}^{2} F(\boldsymbol{\alpha}, \phi) \tilde{\boldsymbol{\alpha}}}{\tilde{\boldsymbol{\alpha}}^{T}\left[\nabla_{\boldsymbol{\alpha}}^{2} F(\boldsymbol{\alpha}, \phi)\right]^{T} \nabla_{\boldsymbol{\alpha}}^{2} F(\boldsymbol{\alpha}, \phi) \tilde{\boldsymbol{\alpha}}}
$$

where $\nabla_{\boldsymbol{\alpha}}^{2} F$ is a symmetric matrix and is often referred to as the Hessian. Note that $\nabla_{\boldsymbol{\alpha}}^{2} F$ includes the variation with respect to $\phi$. Computation of the Hessian is expensive; the cost is proportional to the number of design variables. However, $\nabla_{\boldsymbol{\alpha}}^{2} F \tilde{\boldsymbol{\alpha}}$ can be evaluated relatively easily with finite differences as follows:

$$
\nabla_{\boldsymbol{\alpha}}^{2} F(\boldsymbol{\alpha}, \phi) \tilde{\boldsymbol{\alpha}}=\frac{\nabla_{\boldsymbol{\alpha}} F(\boldsymbol{\alpha}+\bar{\varepsilon} \tilde{\boldsymbol{\alpha}}, \phi+\bar{\varepsilon} \hat{\phi})-\nabla_{\boldsymbol{\alpha}} F(\boldsymbol{\alpha}, \phi)}{\bar{\varepsilon}}
$$

where $\bar{\varepsilon}$ is a trial perturbation. To find the step size, the design variables are first perturbed with an arbitrarily small $\bar{\varepsilon}$, and the new values of the state variables that satisfy the state equations are determined. Next, the new gradient $\nabla_{\boldsymbol{\alpha}} F(\boldsymbol{\alpha}+\bar{\varepsilon} \tilde{\boldsymbol{\alpha}}, \phi+\bar{\varepsilon} \hat{\phi})$ is evaluated, followed by $\nabla_{\boldsymbol{\alpha}}^{2} F(\boldsymbol{\alpha}, \phi) \tilde{\boldsymbol{\alpha}}$. Then, the step size is determined with (22).

\section{The Adjoint Equations}

As stated earlier, the objective of the optimization procedure is to seek a descent direction and a step size in which the design variables can be changed so that the cost function is decreased. To determine the descent direction and the step size, the gradient of the cost function with respect to the design variables $\nabla_{\alpha} F$ (given by (19)) must be evaluated. Efficient and accurate evaluation of the gradient of $F$ is one of the important but difficult steps in any minimization scheme. Equation (19) is not very useful for determining the gradient of $F$ because the efficient and accurate determination of $d \phi / d \boldsymbol{\alpha}$ generally is difficult. However, the adjoint method offers an elegant means for evaluating the gradient easily and accurately. The derivation of the adjoint equations is presented below.

Let the design variables be perturbed such that

$$
\alpha \rightarrow \alpha+\varepsilon \tilde{\boldsymbol{\alpha}}
$$

where $\varepsilon \tilde{\boldsymbol{\alpha}}$ is the change in $\boldsymbol{\alpha}$; $\varepsilon$ and $\tilde{\boldsymbol{\alpha}}$ are the step size (magnitude) and direction, respectively, of the change in $\boldsymbol{\alpha}$. Figure 2 shows the domain after the perturbation, where $\bar{U}$ and $\bar{L}$ denote the upper and lower surfaces, respectively, of 


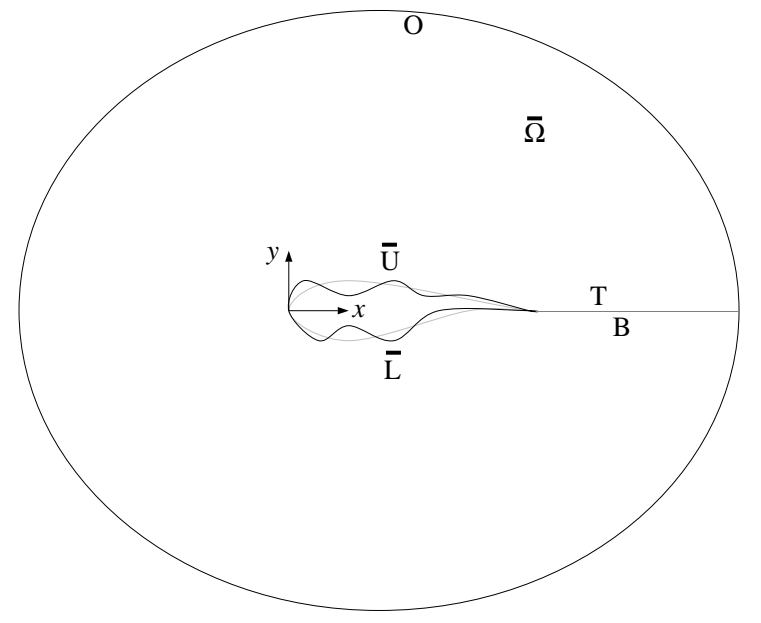

Fig. 2. Domain after perturbation.

the new airfoil and $\bar{\Omega}$ denotes the new domain. The shape of the resulting airfoil $\bar{y}^{\overline{\mathrm{J}}, \overline{\mathrm{L}}}$ and the corresponding potential $\bar{\phi}$ that satisfy the governing equation and its boundary conditions in the new domain can be written as

$$
\bar{y}^{\overline{\mathrm{U}}, \overline{\mathrm{L}}}=y^{\mathrm{U}, \mathrm{L}}+\varepsilon \tilde{y}^{\mathrm{U}, \mathrm{L}}
$$

and

$$
\bar{\phi}=\phi+\varepsilon \tilde{\phi}
$$

where $\varepsilon \tilde{y}$ represents the change in the airfoil shape and $\varepsilon \tilde{\phi}$ represents the corresponding change in the potential. We can show from (13) that

$$
\begin{aligned}
& \tilde{\boldsymbol{\alpha}}^{T} \nabla_{\boldsymbol{\alpha}} F \\
& =\int_{\mathrm{U}+\mathrm{L}} 2\left(\phi-\phi_{0}\right) \phi_{y} \tilde{y} d \sigma+\int_{\mathrm{U}+\mathrm{L}}\left(\phi-\phi_{0}\right)^{2} \frac{y_{x} \tilde{y}_{x}}{1+y_{x}^{2}} d \sigma \\
& +\int_{\mathrm{U}+\mathrm{L}} 2\left(\phi-\phi_{0}\right) \tilde{\phi} d \sigma
\end{aligned}
$$

where $y_{x}=d y / d x$. The objective of this approach is to eliminate those terms that have $\tilde{\phi}$, where

$$
\tilde{\phi}=\frac{d \phi}{d \boldsymbol{\alpha}} \tilde{\boldsymbol{\alpha}}
$$

From the governing equation and its boundary conditions (16), we can show that

$$
\begin{aligned}
\operatorname{div}(\nabla \tilde{\phi}) & =0 & & \text { in } \Omega \\
\nabla \tilde{\phi} \cdot \boldsymbol{n} & =(\tilde{y} \nabla \phi \cdot \boldsymbol{t})_{x} & & \text { on the airfoil } \\
\nabla \tilde{\phi} \cdot \boldsymbol{n} & =\frac{\tilde{\Gamma}}{2 \pi} \nabla \Theta \cdot \boldsymbol{n} & & \text { at the far field } \\
\tilde{\phi}^{\mathrm{T}}-\tilde{\phi}^{\mathrm{B}} & =\tilde{\Gamma} & & \text { along the cut }
\end{aligned}
$$

where

$$
\tilde{\Gamma}=\tilde{\phi}_{t . e .}^{\mathrm{T}}-\tilde{\phi}_{t . e .}^{\mathrm{B}}
$$

and $\boldsymbol{t}$ is the unit tangent.
If we introduce a Lagrange multiplier $\lambda$ and use (29a), then (27) can be written as

$$
\begin{aligned}
& \tilde{\boldsymbol{\alpha}}^{T} \nabla_{\boldsymbol{\alpha}} F \\
& =\int_{\mathrm{U}+\mathrm{L}} 2\left(\phi-\phi_{0}\right) \phi_{y} \tilde{y} d \sigma+\int_{\mathrm{U}+\mathrm{L}}\left(\phi-\phi_{0}\right)^{2} \frac{y_{x} \tilde{y}_{x}}{1+y_{x}^{2}} d \sigma \\
& +\int_{\mathrm{U}+\mathrm{L}} 2\left(\phi-\phi_{0}\right) \tilde{\phi} d \sigma+\iint_{\Omega} \operatorname{div}(\nabla \tilde{\phi}) \lambda d \Omega
\end{aligned}
$$

If we integrate by parts, the last integral can be written as

$$
\begin{aligned}
\iint_{\Omega} \operatorname{div}(\nabla \tilde{\phi}) \lambda d \Omega & =\iint_{\Omega} \operatorname{div}(\nabla \lambda) \tilde{\phi} d \Omega \\
& -\int_{\tau} \lambda(\nabla \tilde{\phi} \cdot \boldsymbol{n}) d \tau+\int_{\tau}(\nabla \lambda \cdot \boldsymbol{n}) \tilde{\phi} d \tau
\end{aligned}
$$

where the unit normal $\boldsymbol{n}$ points into the flow field $\Omega ; d \tau$ is an element on $\tau$, which is the path of integration around the domain $\Omega$ and can be expressed as

$$
\tau=\mathrm{L}+\mathrm{U}+\mathrm{T}+\mathrm{O}+\mathrm{B}
$$

If the integrals along $\tau$ are split into different components and substituted into (31), then we can write

$$
\begin{aligned}
& \tilde{\boldsymbol{\alpha}}^{T} \nabla_{\boldsymbol{\alpha}} F \\
& =\int_{\mathrm{U}+\mathrm{L}} 2\left(\phi-\phi_{0}\right) \phi_{y} \tilde{y} d \sigma+\int_{\mathrm{U}+\mathrm{L}}\left(\phi-\phi_{0}\right)^{2} \frac{y_{x} \tilde{y}_{x}}{1+y_{x}^{2}} d \sigma \\
& +\int_{\mathrm{U}+\mathrm{L}} 2\left(\phi-\phi_{0}\right) \tilde{\phi} d \sigma+\iint_{\Omega} \operatorname{div}(\nabla \lambda) \tilde{\phi} d \Omega \\
& -\int_{\mathrm{U}+\mathrm{L}} \lambda(\nabla \tilde{\phi} \cdot \boldsymbol{n}) d \sigma+\int_{\mathrm{U}+\mathrm{L}}(\nabla \lambda \cdot \boldsymbol{n}) \tilde{\phi} d \sigma \\
& -\int_{\mathrm{T}+\mathrm{B}} \lambda(\nabla \tilde{\phi} \cdot \boldsymbol{n}) d \tau+\int_{\mathrm{T}+\mathrm{B}}(\nabla \lambda \cdot \boldsymbol{n}) \tilde{\phi} d \tau \\
& -\int_{\mathrm{O}} \lambda(\nabla \tilde{\phi} \cdot \boldsymbol{n}) d \tau \quad+\int_{\mathrm{O}}(\nabla \lambda \cdot \boldsymbol{n}) \tilde{\phi} d \tau
\end{aligned}
$$

Because $\nabla \tilde{\phi}$ is continuous across the cut and $\boldsymbol{n}$ points in opposite directions along the top and bottom boundaries of the cut, we can write

$$
\int_{\mathrm{T}+\mathrm{B}} \lambda(\nabla \tilde{\phi} \cdot \boldsymbol{n}) d \tau=\int_{\mathrm{Cut}}\left(\lambda^{\mathrm{T}}-\lambda^{\mathrm{B}}\right)(\nabla \tilde{\phi} \cdot \boldsymbol{n}) d \tau
$$

If we assume that $\nabla \lambda$ is continuous across the cut, then we can write

$$
\int_{\mathrm{T}+\mathrm{B}}(\nabla \lambda \cdot \boldsymbol{n}) \tilde{\phi} d \tau=\tilde{\Gamma} \int_{\mathrm{Cut}} \nabla \lambda \cdot \boldsymbol{n} d \tau
$$


If we use (35), (36), and (29b-d), then equation (34) can be written as

$$
\begin{aligned}
& \tilde{\boldsymbol{\alpha}}^{T} \nabla_{\boldsymbol{\alpha}} F \\
& =\int_{\mathrm{U}+\mathrm{L}} 2\left(\phi-\phi_{0}\right) \phi_{y} \tilde{y} d \sigma+\int_{\mathrm{U}+\mathrm{L}}\left(\phi-\phi_{0}\right)^{2} \frac{y_{x} \tilde{y}_{x}}{1+y_{x}^{2}} d \sigma \\
& +\int_{\mathrm{U}+\mathrm{L}} 2\left(\phi-\phi_{0}\right) \tilde{\phi} d \sigma+\iint_{\Omega} \operatorname{div}(\nabla \lambda) \tilde{\phi} d \Omega \\
& -\int_{\mathrm{U}+\mathrm{L}} \lambda(\tilde{y} \nabla \phi \cdot \boldsymbol{t})_{x} d \sigma+\int_{\mathrm{U}+\mathrm{L}}(\nabla \lambda \cdot \boldsymbol{n}) \tilde{\phi} d \sigma \\
& -\int_{\mathrm{Cut}}\left(\lambda^{\mathrm{T}}-\lambda^{\mathrm{B}}\right)(\nabla \tilde{\phi} \cdot \boldsymbol{n}) d \tau+\tilde{\Gamma} \int_{\mathrm{Cut}} \nabla \lambda \cdot \boldsymbol{n} d \tau \\
& -\frac{\tilde{\Gamma}}{2 \pi} \int_{\mathrm{O}} \lambda(\nabla \Theta \cdot \boldsymbol{n}) d \tau+\int_{\mathrm{O}}(\nabla \lambda \cdot \boldsymbol{n}) \tilde{\phi} d \tau
\end{aligned}
$$

If we substitute for $\tilde{\Gamma}$ from (30) and rearrange, then (37) becomes

$$
\begin{aligned}
& \tilde{\boldsymbol{\alpha}}^{T} \nabla_{\boldsymbol{\alpha}} F \\
& =\int_{\mathrm{U}+\mathrm{L}} 2\left(\phi-\phi_{0}\right) \phi_{y} \tilde{y} d \sigma+\int_{\mathrm{U}+\mathrm{L}}\left(\phi-\phi_{0}\right)^{2} \frac{y_{x} \tilde{y}_{x}}{1+y_{x}^{2}} d \sigma \\
& -\int_{\mathrm{U}+\mathrm{L}} \lambda(\tilde{y} \nabla \phi \cdot \boldsymbol{t})_{x} d \sigma+\iint_{\Omega} \operatorname{div}(\nabla \lambda) \tilde{\phi} d \Omega \\
& +\int_{\mathrm{U}+\mathrm{L}}\left[\nabla \lambda \cdot \boldsymbol{n}+2\left(\phi-\phi_{0}\right)\right] \tilde{\phi} d \sigma \\
& \left.+\tilde{\phi}_{t . e .}^{\mathrm{U}}-\tilde{\phi}_{t . e .}^{\mathrm{L}}\right)\left(\int_{\mathrm{Cut}} \nabla \lambda \cdot \boldsymbol{n} d \tau-\frac{1}{2 \pi} \int_{\mathrm{O}} \lambda \nabla \Theta \cdot \boldsymbol{n} d \tau\right) \\
& +\int_{0}(\nabla \lambda \cdot \boldsymbol{n}) \tilde{\phi} d \tau-\int_{\mathrm{Cut}}\left(\lambda^{\mathrm{T}}-\lambda^{\mathrm{B}}\right)(\nabla \tilde{\phi} \cdot \boldsymbol{n}) d \tau
\end{aligned}
$$

We choose $\lambda$ such that

$$
\begin{aligned}
\operatorname{div}(\nabla \lambda) & =0 & & \text { in } \Omega \\
\nabla \lambda \cdot \boldsymbol{n}+2\left(\phi-\phi_{0}\right)-\Upsilon \delta\left(x-x_{t . e .}\right) & =0 & & \text { on } \mathrm{L} \\
\nabla \lambda \cdot \boldsymbol{n}+2\left(\phi-\phi_{0}\right)+\Upsilon \delta\left(x-x_{t . e .}\right) & =0 & & \text { on } \mathrm{U} \\
\nabla \lambda \cdot \boldsymbol{n} & =0 & & \text { in the far field } \\
\lambda^{\mathrm{T}}-\lambda^{\mathrm{B}} & =0 & & \text { along the cut }
\end{aligned}
$$

where

$$
\Upsilon=\int_{\mathrm{Cut}} \nabla \lambda \cdot \boldsymbol{n} d \tau-\frac{1}{2 \pi} \int_{\mathrm{O}} \lambda \nabla \Theta \cdot \boldsymbol{n} d \tau
$$

and $\delta$ denotes the Dirac delta function. Equations (39) are the adjoint equation and its boundary conditions (also called the costate equations). These equations are similar to the linearized state equations. The size of the system is the same as the size of the state equations and can be solved with the same technique used to solve the state equations.
Because $\operatorname{div}(\nabla \lambda)=0$ in $\Omega$, we obtain the following from the divergence theorem:

$$
\int_{\tau} \nabla \lambda \cdot \boldsymbol{n} d \tau=0
$$

Therefore, so that (39) has a solution, we can show that

$$
\int_{\mathrm{U}+\mathrm{L}}\left(\phi-\phi_{0}\right) d \sigma \equiv 0
$$

Equation (16) clearly shows that a constant can be added to $\phi$. We can choose this constant $\phi_{c}$ such that

$$
\int_{\mathrm{U}+\mathrm{L}}\left(\phi+\phi_{c}-\phi_{0}\right) d \sigma=0
$$

Therefore,

$$
\phi_{c}=-\frac{\int_{\mathrm{U}+\mathrm{L}}\left(\phi-\phi_{0}\right) d \sigma}{\int_{\mathrm{U}+\mathrm{L}} d \sigma}
$$

\section{The Gradient of $F$}

If (39) is substituted into (38), then it reduces to

$$
\begin{aligned}
& \tilde{\boldsymbol{\alpha}}^{T} \nabla_{\boldsymbol{\alpha}} F \\
& =\int_{\mathrm{U}+\mathrm{L}} 2\left(\phi-\phi_{0}\right) \phi_{y} \tilde{y} d \sigma+\int_{\mathrm{U}+\mathrm{L}}\left(\phi-\phi_{0}\right)^{2} \frac{y_{x} \tilde{y}_{x}}{1+y_{x}^{2}} d \sigma \\
& -\int_{\mathrm{U}+\mathrm{L}} \lambda(\tilde{y} \nabla \phi \cdot \boldsymbol{t})_{x} d \sigma
\end{aligned}
$$

If we integrate the last integral by parts, then we get

$$
\begin{aligned}
& -\int_{\mathrm{U}+\mathrm{L}} \lambda(\tilde{y} \nabla \phi \cdot \boldsymbol{t})_{x} d \sigma \\
& =\int_{\mathrm{U}+\mathrm{L}} \tilde{y} \nabla \phi \cdot \boldsymbol{t} \lambda_{x} d \sigma+\int_{\mathrm{U}+\mathrm{L}} \tilde{y} \nabla \phi \cdot \boldsymbol{t} \lambda \frac{y_{x} y_{x x}}{1+y_{x}^{2}} d \sigma
\end{aligned}
$$

If (46) is substituted into (45), then we can write

$$
\begin{aligned}
& \tilde{\boldsymbol{\alpha}}^{T} \nabla_{\boldsymbol{\alpha}} F \\
& =\int_{\mathrm{U}+\mathrm{L}} 2\left(\phi-\phi_{0}\right) \phi_{y} \tilde{y} d \sigma+\int_{\mathrm{U}+\mathrm{L}}\left(\phi-\phi_{0}\right)^{2} \frac{y_{x} \tilde{y}_{x}}{1+y_{x}^{2}} d \sigma \\
& +\int_{\mathrm{U}+\mathrm{L}} \tilde{y} \nabla \phi \cdot \boldsymbol{t} \lambda_{x} d \sigma+\int_{\mathrm{U}+\mathrm{L}} \tilde{y} \nabla \phi \cdot \boldsymbol{t} \lambda \frac{y_{x} y_{x x}}{1+y_{x}^{2}} d \sigma
\end{aligned}
$$

If we substitute for $\tilde{y}$ from (11), then (47) can be written as

$$
\tilde{\boldsymbol{\alpha}}^{T} \nabla_{\boldsymbol{\alpha}} F=\sum_{k=1}^{K} \tilde{\alpha}_{k}^{\mathrm{U}} \boldsymbol{\mu}_{k}^{\mathrm{U}}+\sum_{k=1}^{K} \tilde{\alpha}_{k}^{\mathrm{L}} \boldsymbol{\mu}_{k}^{\mathrm{L}}
$$


where

$$
\begin{aligned}
\mu_{k}^{\mathrm{U}, \mathrm{L}} & =\int_{\mathrm{U}, \mathrm{L}}\left(\phi-\phi_{0}\right)^{2} \frac{y_{x}}{1+y_{x}^{2}}\left(f_{k}\right)_{x} d \sigma \\
& +\int_{\mathrm{U}, \mathrm{L}}\left[2\left(\phi-\phi_{0}\right) \phi_{y}+\nabla \phi \cdot \boldsymbol{t} \lambda_{x}+\nabla \phi \cdot \boldsymbol{t} \lambda \frac{y_{x} y_{x x}}{1+y_{x}^{2}}\right] f_{k} d \sigma
\end{aligned}
$$

Equations (49) are the components of the gradient of $F$. When $\phi$ satisfies the state equations (16) and $\lambda$ satisfies the costate equations (39), then the components of the gradient of $F$ can be evaluated with (49). Because $\nabla_{\alpha} F=0$ at the minimum, we can clearly see that

$$
\left.\begin{array}{l}
\mu_{k}^{\mathrm{U}}=0 \\
\mu_{k}^{L}=0
\end{array}\right\} \quad \text { for } k=1,2, \ldots K
$$

\section{A Design Strategy}

Figure 3 shows a typical design strategy. In this process, at some initial conditions the state and adjoint equations are solved, and the gradient of $F$ is computed. If the gradient is equal to 0 , then a minimum has been reached and the iteration is terminated; otherwise, the new descent direction $\tilde{\boldsymbol{\alpha}}$ and

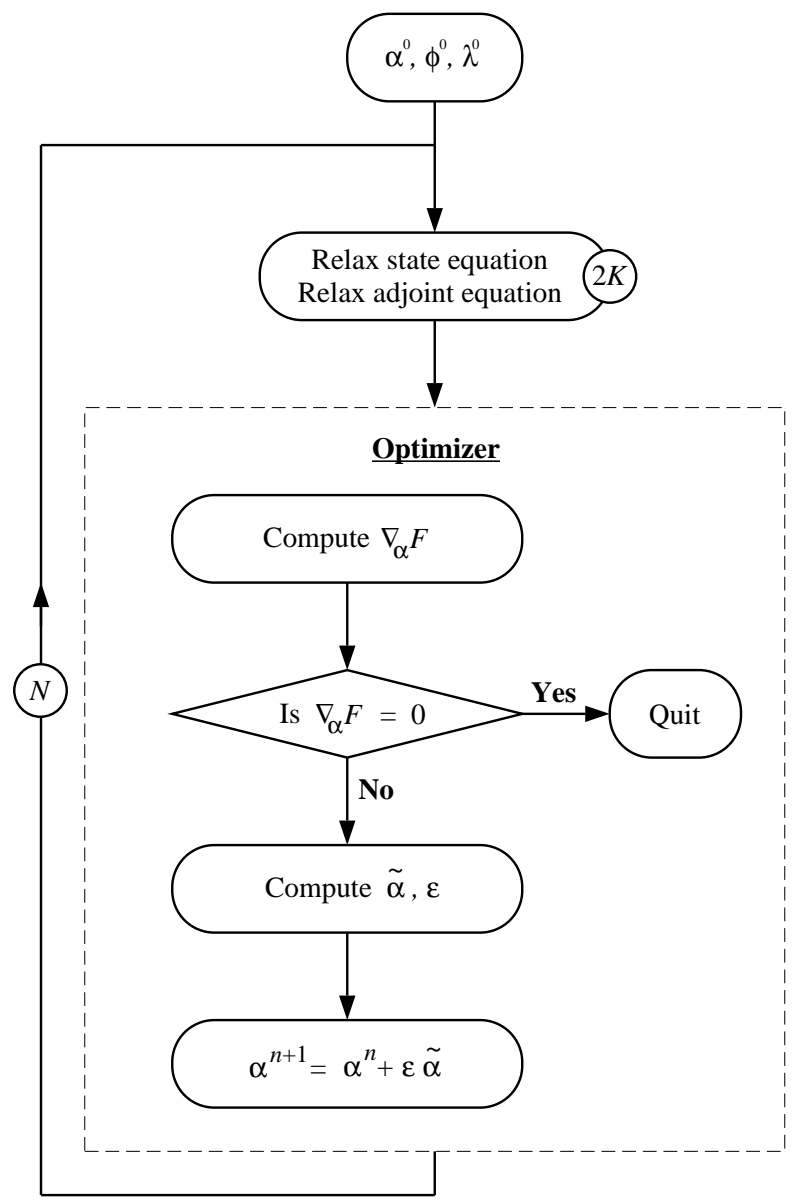

Fig. 3. A design strategy flowchart. the step size $\varepsilon$ are computed, and the design variables are updated. The iteration is repeated until the gradient vanishes. The cost of this strategy can be estimated as follows. Let the cost of solving the state equations be equal to $K$. The cost of solving the adjoint equation is at most equal to $K$. Let the number of design iterations required be $N$. Therefore, the total cost of doing the optimal design is approximately $2 K N$ with $N$, at best, equal to the number of design variables. In practice, especially for nonlinear problems, $N$ is many times the number of design variables. A factor of 100 is not unrealistic. One way to bring the total design cost down is to reduce the magnitude of $K$. One of the most practical and proven ways of achieving this is by using multigrid. Here, a multigrid scheme is used to relax the state and adjoint equations. At the end of one or several multigrid cycles, the optimizer is called and the design variables are updated. In this process, the design variables are updated only on the finest grid. A schematic of this strategy is shown in Fig. 4.

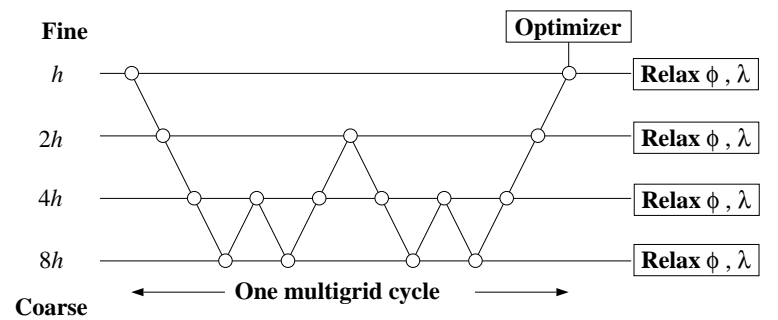

Fig. 4. A multigrid strategy.

\section{The One-Shot Method}

The One-Shot method goes one step further by embedding the design process within the multigrid cycles. This method essentially makes $N=1$. Thus, the cost of optimal design is approximately equal to $2 K$. In this method, high efficiency is obtained by exploiting two key phenomena: the ability of multigrid to efficiently reduce high-frequency components of the error due to a perturbation and the nature of propagation of perturbations in a flow field. These phenomena are explained below.

\section{Multigrid Efficiency}

In any relaxation (smoothing) process, the highfrequency error components of the space discretization operator of the differential equation under consideration are generally damped in a few iterations. The low-frequency components are the slowest to be damped. Consider a onedimensional domain of length $L$ discretized into $N$ cells of uniform grid spacing $h=L / N$, where the grid index ranges from 0 to $N$. This grid will be referred to as the $h$ grid. If we assume periodic boundary conditions, then the error at the $n$th grid point can be written in Fourier series as

$$
\epsilon_{n}=\sum_{j=-N}^{N} A_{j} e^{i \theta_{j} n}
$$


where $A_{j}$ is the amplitude of the $j$ th harmonic and $i=\sqrt{-1}$. The phase angle $\theta$ can be written as

$$
\theta_{j}=\frac{j \pi}{N}
$$

The phase angle covers the domain $(-\pi, \pi)$ in increments of $\pi / N$. The value $|\theta|=\pi$ corresponds to the highest frequency that is visible on this grid, namely the frequency of wavelength $2 h$. If a coarse grid ( $H$ grid) is constructed by removing every other grid point of the $h$ grid, then the highest frequency that is visible on this grid corresponds to $|\theta|=\pi / 2$ (i.e., the frequency of wavelength $4 h \equiv 2 H$ ). Therefore, the frequencies that correspond to $\pi / 2<|\theta| \leq \pi$ and are visible on the $h$ grid cannot be represented on the $H$ grid. These frequencies are considered to be high frequencies on the $h$ grid, and the relaxation scheme can damp these frequencies in a few iterations. The remaining frequencies in the spectrum, which correspond to $0 \leq|\theta| \leq \pi / 2$ and are well represented on the $H$ grid, are referred to as low frequencies on the $h$ grid. The frequencies that are visible on the $H$ grid can also be separated into high and low frequencies, based on how well they are represented by the next coarsest grid. The high frequencies that correspond to the $H$ grid can be damped quickly by a few iterations of the relaxation scheme on this grid.

In the multigrid method, ${ }^{17,18}$ high efficiency is obtained by relaxing the discretized equation on successively coarser grids, where the high-frequency error components that correspond to each grid are damped efficiently. In the design process, high efficiency is obtained by changing only those design variables that produce high-frequency perturbations in the flow field on any grid. Therefore, the basic premise of the One Shot method, on any grid, is to make changes in the design variables that produce high-frequency perturbations in the flow field.

\section{The Effect of Airfoil Perturbation on the Flow Field}

The other phenomenon that is exploited by the OneShot method has to do with the way in which a disturbance is propagated in a flow field. In a subsonic flow, for example, a smooth perturbation is propagated through the entire flow field and a high-frequency perturbation is felt only in a small neighborhood around the source of the perturbation. That is, high-frequency components of the perturbation decay rapidly away from the source. This phenomenon is illustrated in the following analysis.

Consider the small-disturbance potential equation in the half-space $0 \leq y<\infty,-\infty<x<\infty$. If the flow is incompressible, the governing equation is

$$
\nabla^{2} \phi=0
$$

and the boundary condition applied at $y=0$ is

$$
\frac{\partial \phi}{\partial y}=\frac{\partial f}{\partial x}
$$

where $f(x)$ is the shape of the boundary over which the flow must be determined. If $\phi+\tilde{\phi}$ is the potential due to a change in shape to $f+\tilde{f}$, the governing equation for change in potential $\tilde{\phi}$ is

$$
\nabla^{2} \tilde{\phi}=0
$$

and the boundary condition at $y=0$ is

$$
\frac{\partial \tilde{\phi}}{\partial y}=\frac{\partial \tilde{f}}{\partial x}
$$

Let

$$
\frac{\partial \tilde{f}}{\partial x}=e^{i \omega x}
$$

where $\omega$ is the frequency of the perturbation. A solution to the governing equation (55) that satisfies the boundary condition is

$$
\tilde{\phi}=e^{-|\omega| y} e^{i \omega x}
$$

The magnitude of $\tilde{\phi}$ is

$$
|\tilde{\phi}|=e^{-|\omega| y}
$$

Figure 5, which is the plot of (59) for a few select frequencies,

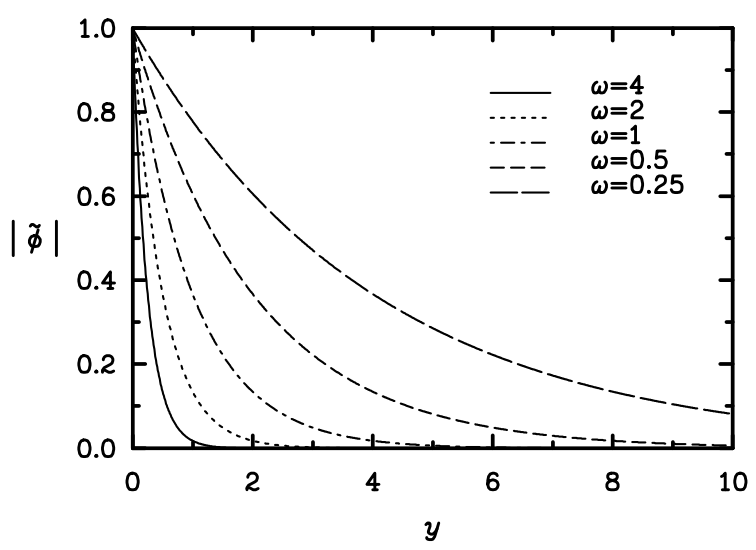

Fig. 5. $|\tilde{\phi}|$ versus $y$.

shows that the region where $\tilde{\phi}$ is large becomes thinner as the frequency increases. Let $y^{*}$ be a location where $\tilde{\phi}$ is less than some small $\varepsilon$. That is,

$$
\left|\tilde{\phi}\left(\omega, y^{*}\right)\right|<\varepsilon=e^{-\beta}
$$

If we substitute for $\tilde{\phi}$, then

$$
e^{-\omega \mid y^{*}}=e^{-\beta}
$$

Therefore,

$$
y^{*}=\frac{-\ln (\varepsilon)}{|\omega|}
$$

Equation (62) clearly shows that as the frequency of the perturbation $\omega$ increases $y^{*}$ decreases. Table 1 shows $y^{*}$ 
for a few select frequencies when $\varepsilon=10^{-4}$. For the discrete

Table 1. $y^{*}$ Versus $\omega$

\begin{tabular}{|c|c|c|c|c|c|}
\hline$\omega$ & $1 / 4$ & $1 / 2$ & 1 & 2 & 4 \\
\hline$y^{*}$ & 27.6 & 13.8 & 6.9 & 3.5 & 1.7 \\
\hline
\end{tabular}

problem, (59) can be written as

$$
|\tilde{\phi}|=e^{-(|\omega| h)(y / h)}=e^{-|\theta|(j-1)} \quad(j=1,2, \ldots J+1)
$$

where $\pi / J \leq \theta \leq \pi$ is the frequency scaled to the grid spacing $h$. Figure 6 shows the response to different frequencies for the discrete problem. Table 2 shows the grid location $j^{*}$, beyond which $|\tilde{\phi}| \leq 10^{-4}$. It shows that the high-frequency perturbations are significantly damped by about the fifth grid point ( $j=0$ is the first grid point).

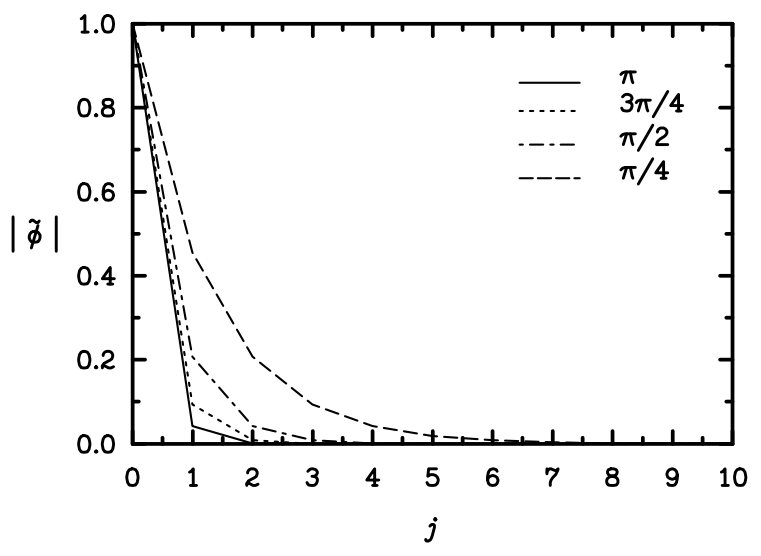

Fig. 6. $|\tilde{\phi}|$ versus $j$.

Table 2. $j^{*}$ Versus $\theta$

\begin{tabular}{|c|c|c|c|c|}
\hline$\theta$ & $\pi / 4$ & $\pi / 2$ & $3 \pi / 4$ & $\pi$ \\
\hline$j^{*}$ & 8.8 & 4.4 & 2.9 & 2.2 \\
\hline
\end{tabular}

In the One-Shot method, a shape function is perturbed on a grid where it produces high-frequency error components. These errors penetrate only a small distance into the flow field. Hence, they can be quickly damped by a few relaxations of the discrete equations in a small neighborhood around the airfoil.

\section{The Shape Functions}

As presented earlier (section 3), the airfoil is represented as follows:

$$
y^{\mathrm{U}, \mathrm{L}}=\sum_{k=1}^{K} \alpha_{k}^{\mathrm{U}, \mathrm{L}} f_{k}(x)
$$

where $\alpha_{k}^{\mathrm{U}}$ and $\alpha_{k}^{\mathrm{L}}$ are the design variables and $f_{k}$ are the shape functions. As explained in the previous two sections, to obtain high design efficiency, the changes in the design variables on a grid should produce nonsmooth (high-frequency) perturbations in the flow field. This is achieved by using a set of orthonormal functions as shape functions. Orthonormal functions are increasingly oscillatory. Each of them is assigned to a grid where a change in the amplitudes causes nonsmooth perturbations in the flow field. Often, basis functions that correspond to some known airfoil shape must be used. If these functions are not orthonormal, then the corresponding orthonormal functions can be determined by a Gram-Schmidt process. A Gram-Schmidt procedure for orthonormalization can be developed with the property of orthonormal functions, namely,

$$
\begin{gathered}
\int_{0}^{1} f_{m}(x) f_{n}(x) d x=0 \quad(m \neq n) \\
\int_{0}^{1} f_{m}^{2}(x) d x=1
\end{gathered}
$$

Let $g_{k}(x)$ be the functions that are not orthonormal. First, the orthogonal set $\bar{f}_{k}(x)$ is found from the following relations:

$$
\begin{aligned}
\bar{f}_{1}(x) & =g_{1}(x) \\
\bar{f}_{2}(x) & =g_{2}(x)+a_{21} \bar{f}_{1}(x) \\
\cdot & \\
\cdot & \\
\bar{f}_{k}(x) & =g_{k}(x)+\sum_{m=1}^{k-1} a_{k m} \bar{f}_{m}(x)
\end{aligned}
$$

where

$$
a_{k m}=-\frac{\int_{0}^{1} g_{k}(x) \bar{f}_{m}(x) d x}{\int_{0}^{1} \bar{f}_{m}^{2}(x) d x}
$$

Finally, the orthonormal functions are found by normalizing $\bar{f}_{k}(x)$ as follows:

$$
f_{k}=\frac{\bar{f}_{k}(x)}{\sqrt{\int_{0}^{1} \bar{f}_{k}^{2}(x) d x}}
$$

The Gram-Schmidt process described above can be programmed in symbolic language to find the expressions for 
$f_{k}$, or it can be implemented by numerical integration, in which case the shape functions are defined as an array of numbers.

As an example, consider the NACA 0012 airfoil, which is defined by

$$
\begin{aligned}
y^{\mathrm{U}} & =\sum_{k=1}^{4} \beta_{k} g_{k}(x) \quad(0 \leq x \leq 1) \\
y^{\mathrm{L}} & =-y^{\mathrm{U}}
\end{aligned}
$$

where $\beta_{k}$ and $g_{k}$ are given in Table 3. The NACA 0012 shape has been slightly modified to ensure that it closes at the trailing edge. The same shape can be expressed in terms of the orthonormal functions as

$$
\begin{aligned}
y^{\mathrm{U}} & =\sum_{k=1}^{4} \alpha_{k} f_{k}(x) \quad(0 \leq x \leq 1) \\
y^{\mathrm{L}} & =-y^{\mathrm{U}}
\end{aligned}
$$

where the orthonormal functions $f_{k}$ of the basis functions and their corresponding amplitudes $\alpha_{k}$ are given in Table 4 . The orthonormal shape functions are shown in Fig. 7. Note that the number of zeros in $f_{k}$ is equal to $k+1$.

Table 3. Shape Functions and Amplitudes of NACA 0012

\begin{tabular}{|c|c|c|}
\hline$k$ & $\beta_{k}$ & $g_{k}$ \\
\hline \hline 1 & 0.17814 & $\sqrt{x}-x$ \\
\hline 2 & 0.10128 & $x(1-x)$ \\
\hline 3 & -0.10968 & $x^{2}(1-x)$ \\
\hline 4 & 0.06090 & $x^{3}(1-x)$ \\
\hline
\end{tabular}

Table 4. Orthonormal Shape Functions and Amplitudes of NACA 0012

\begin{tabular}{|c|c|c|}
\hline$k$ & $\alpha_{k} \times 10^{4}$ & $f_{k}$ \\
\hline \hline 1 & 439.474 & $5.47723 g_{1}$ \\
\hline 2 & 28.2339 & $14.7573\left(g_{2}-0.928571 g_{1}\right)$ \\
\hline 3 & -5.85699 & $54.7884\left(g_{3}-0.901236 g_{2}\right.$ \\
& & $\left.+0.432099 g_{1}\right)$ \\
\hline 4 & 2.85283 & $\left.+0.504011 g_{2}-0.164439 g_{1}\right)$ \\
& & \\
\hline
\end{tabular}

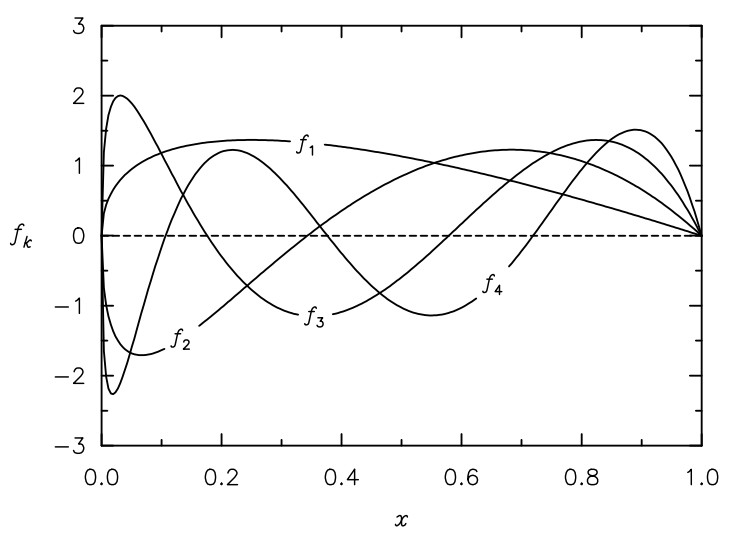

Fig. 7. Orthonormal shape functions of NACA 0012 airfoil.

\section{The One-Shot Design Strategy}

In the One-Shot method, the optimizer is embedded within the multigrid cycle as shown in Fig. 8. The design variables are updated on a level where the corresponding shape functions produce high-frequency error components. In general, the low-frequency shape functions are updated on coarse levels, and higher frequency functions are updated on finer grids. For example, the design variables $\alpha_{1}^{\mathrm{U}}$ and $\alpha_{1}^{\mathrm{L}}$ are updated on the coarsest grid $8 h$; $\alpha_{1}^{\mathrm{U}}, \alpha_{2}^{\mathrm{U}}, \alpha_{3}^{\mathrm{U}}, \alpha_{4}^{\mathrm{U}}, \alpha_{1}^{\mathrm{L}}, \alpha_{2}^{\mathrm{L}}, \alpha_{3}^{\mathrm{L}}$, and $\alpha_{4}^{\mathrm{L}}$ are updated on the next finest grid $4 h$. Some overlap of the design variables is permitted. Thus, $\alpha_{1}^{\mathrm{U}}$ and $\alpha_{1}^{\mathrm{L}}$ are updated on grid $4 h$ also. None of the design variables are updated on the finest grid $h$. The cost of solving the state or the adjoint equations on a coarse grid is only one-fourth of the cost of solving them on the next finest grid. Because the shape functions are perturbed only on levels where they generate high-frequency errors, a local relaxation around the airfoil is sufficient to damp out the errors, which reduces computing costs. Therefore, the overall cost of the design is dominated by the cost required to solve the state and adjoint equations on the finest grid. The total cost of the design process is approximately two to three times that of one analysis.

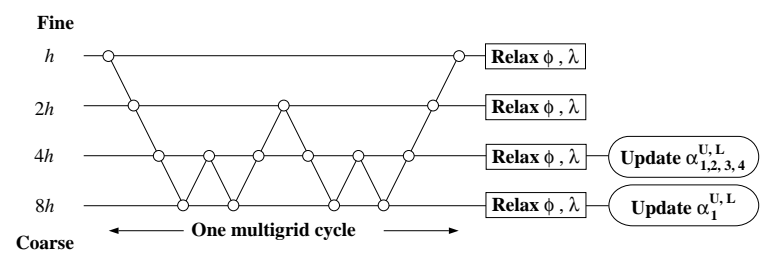

Fig. 8. The One-Shot strategy. 


\section{The Discretization and Solution Procedure}

The State Equations. The computational domain is discretized with an $\mathrm{O}$ type of grid. The governing equation and its boundary conditions cast in curvilinear coordinates are discretized with the finite-volume approach. The GaussSeidel line-relaxation scheme is used to form the tridiagonal systems of equations in both curvilinear coordinate directions. These systems are solved with the Thomas algorithm. Note that the tridiagonal system is periodic in the direction that is around the airfoil. A FAS multigrid scheme is used to accelerate the convergence rate of the solution. The FMG process is used to obtain a good initial solution on the finest grid.

The Adjoint Equations. The adjoint equations are discretized and solved in the same manner as the state equations. As in the case of the state equations, a FAS multigrid scheme and the FMG process are used to accelerate the convergence rate of the solution.

The Gradient of $F$. The gradient of the cost function involves only quantities on the airfoil. These quantities are discretized in a manner that is consistent with the discretization of the state and adjoint equations. The gradient is transferred to the coarse grid in a FAS manner.

Updating the Grid. During the design process, the grid is updated by moving only the grid points close to the airfoil and linearly decaying the change at the airfoil in this neighborhood. The outer boundary of this region is determined as follows. Let

$$
y_{\max }=\eta \max (\varepsilon \tilde{y})
$$

where $\eta$ is an arbitrary constant; $\eta=10$ in this study. Among the grid lines that go around the airfoil, the one that is nearest to the $y_{\max }$ location is taken to be the outer boundary of the region within which the grids are changed. The entire grid is regenerated at the beginning of each FMG stage also. With this approach, by the time the FMG process reaches the finest grid, only a few lines around the airfoil must be moved.

\section{THE RESULTS}

\section{Test Case 1.}

As our first test problem, we recover the NACA 0012 airfoil shape using the potential distribution obtained from the analysis of NACA 0012 at an angle of attack of $0^{\circ}$ and $M_{\infty}=0$ as the target potential $\phi_{0}$. Figure 9 shows the computed $C_{p}$ distribution obtained from the analysis run. A five-level $\mathrm{W}$-cycle multigrid with $128 \times 64$ cells on the finest grid was used. The FMG process was used to obtain a good initial approximation for the finest grid. The analysis converged to machine zero $\left(<10^{-10}\right)$ in 10 multigrid cycles.

The design run was similar to the analysis run. During the design process, both the state and costate equations were relaxed at any multigrid level. The shape functions used were the orthonormal functions based on the NACA 0012 shape functions. The design variables were distributed such that on
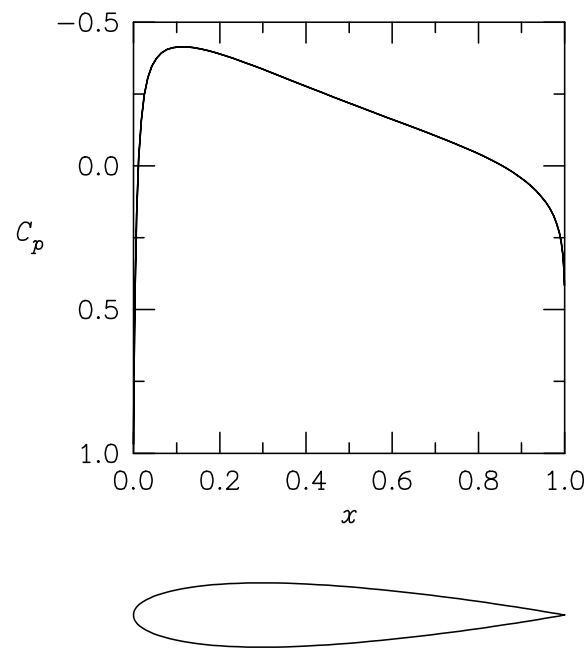

Fig. 9. Computed $C_{p}$ distribution for NACA 0012.

the coarsest level $(8 \times 4)$ only $\alpha_{1}^{\mathrm{U}}$ and $\alpha_{1}^{\mathrm{L}}$ were updated. On the next finest level $(16 \times 8)$, all the design variables $\left(\alpha_{1,2,3,4}^{\mathrm{U}, \mathrm{L}}\right)$ were updated. None of the design variables were updated on the next three levels, including the finest level. Thus, most of the design overhead was limited to the two coarsest grids. The FMG process was used to obtain a good initial approximation of the shape for the finest grid. Figure 10 shows the results of this run. The residuals of the state and costate equations and the gradient of the cost function reached machine zero in 12 multigrid cycles. The cost function at convergence was equal to $3 \times 10^{-13}$, which indicates that NACA 0012 was indeed recovered.
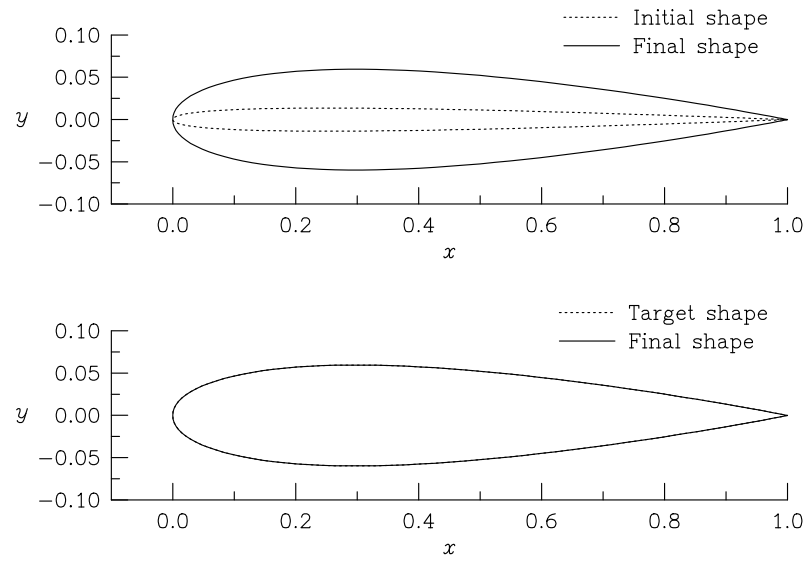

Fig. 10. Test case 1.

Test Case 2.

For test case 2, we selected the airfoil FX 60-126/1, a cambered airfoil whose coordinates are tabulated in Ref. 19. Figure 11 shows the $C_{p}$ distribution for this airfoil at an angle of attack of $0^{\circ}$ and $M_{\infty}=0$. This airfoil is not smooth, which is reflected in the computed $C_{p}$ distribution. Using this solution as the target, we tried to recover the shape with the NACA 0012 shape functions. Figure 12 shows the 
resulting shape. Although the designed shape did not fall right on top of the target shape, the residuals of the state and costate equations and the gradient of the cost function reached machine zero, which indicates that a minimum was reached. The cost function reached a value of $6 \times 10^{-9}$.

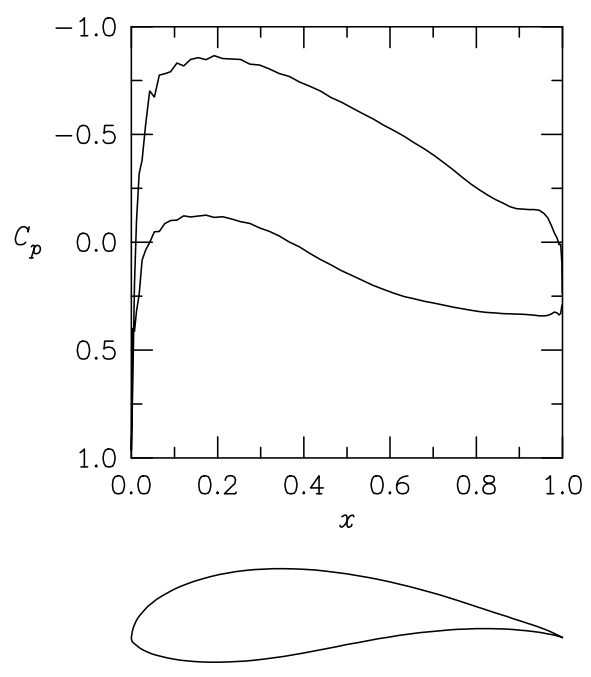

Fig. 11. Computed $C_{p}$ distribution for FX 60-126/1.
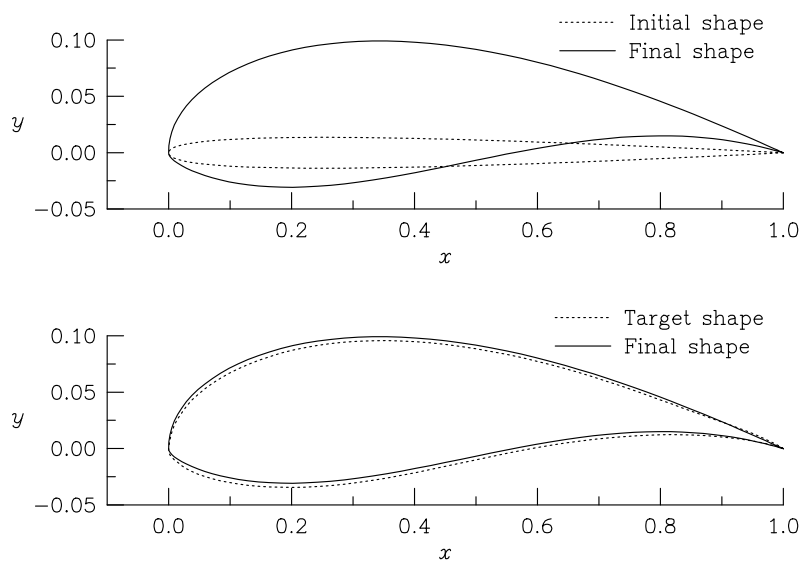

Fig. 12. Test case 2 .

Next, an experiment was done to see how well the FX 60-126/1 airfoil can be represented with the NACA 0012 shape functions. Figure 13 shows the result. The NACA 0012 shape functions clearly do a good job everywhere except near the trailing edge. The reason that the optimum shape in the previous experiment does not correspond to the shape obtained from the shape fitting is not clear; one reason may be the poor quality of the grid because the airfoil is not smooth.

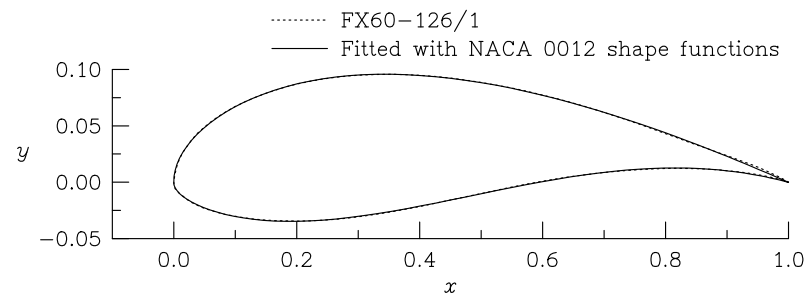

Fig. 13. Shape fitting with NACA 0012 shape functions.

Test Case 3.

A third test was done; in this case, the fitted airfoil was used to generate the target potential. This shape is very close to the FX 60-126/1 airfoil and is smooth because it is based on smooth shape functions. The result of the design is shown in fig. 14. As expected, the final shape fell on top of the target shape. The residuals of the state and costate equations and the gradient of the cost function are shown in fig. 15 .

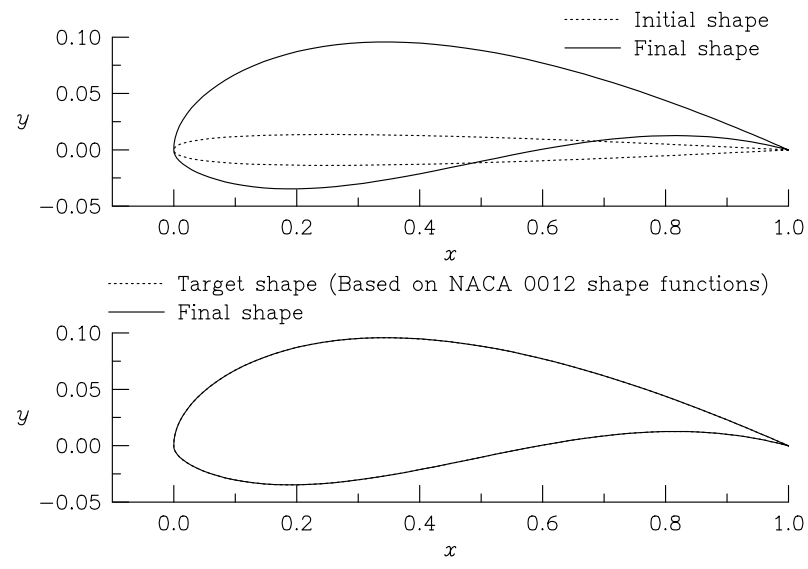

Fig. 14. Test case 3 .

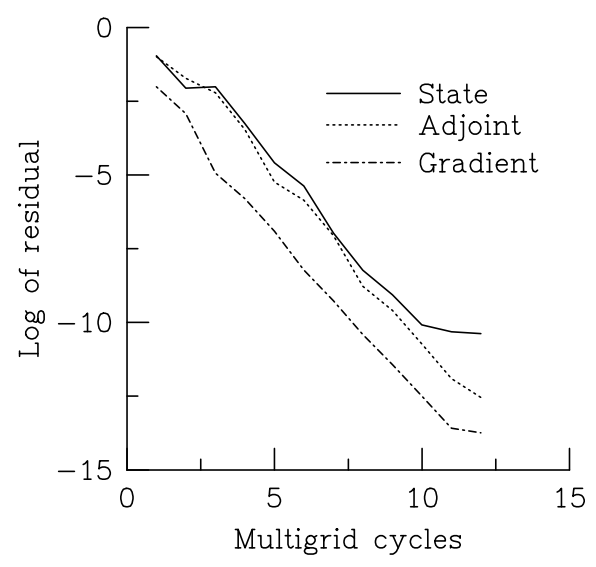

Fig. 15. Convergence history. 


\section{The Efficiency of One-Shot Method}

Finally, the performance of the One-Shot method with respect to pure analysis is presented. The efficiency of a design method is defined as the ratio of the central processing unit (CPU) time that is required for the complete design process $t_{D}$ to the CPU time that is required to do a single analysis $t_{A}$. Figure 16 shows this ratio $t_{D} / t_{A}$ plotted against the number of grid cells for the last test case. The figure shows that as the grid become finer the cost of design drops in comparison with the cost of one analysis. For the finest grid considered here, this ratio dropped below 4 . The efficiencies for the other cases were similar.

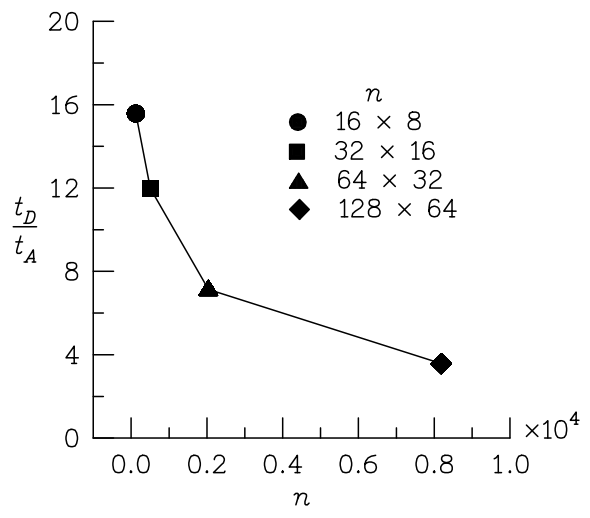

Fig. 16. Efficiency of the One-Shot method.

\section{Concluding Remarks}

An efficient method for the design of optimal airfoil shapes has been presented in this paper. This method brings the cost of the design process to the same order as that of the analysis problem. It offers significant potential in the design of optimal aircraft configurations at a reasonable computer cost. However, much work is still required before practical use can be made of this method.

\section{Acknowledgments}

The authors would like to thank Jonay Campbell, MHSI, for her help in editing the manuscript. The first and second authors were supported by the National Aeronautics and Space Administration (NASA) under contract numbers NAS1-19672 and NAS1-19480, respectively.

\section{References}

1 M. J. Lighthill, "A new method of two dimensional aerodynamic design," ARC Rand M 2112, 1945.

2 F. Bauer, P. Garabedian, and D. Korn, A Theory of Supercritical Wing Sections with Computer Programs and Examples. Springer-Verlag, 1972.

3 J. L. Steger and J. M. Klineberg, "A finite difference method for transonic airfoil design," AIAA J., Vol. 11, No. 5, 1973, pp. 628-635.
4 M. Giles, M. Drela, and W. T. Thompkins, "Newton solution of direct and inverse transonic euler equations," in AIAA 7th Computational Fluid Dynamics Conference., 1985, pp. 394-402.

5 G. Volpe and R. E. Melnik, "The design of transonic aerofoils by a well posed inverse method," Int. J. Numerical Methods in Engineering, Vol. 22, 1986, pp. 341-361.

6 L. A. Carlson, R. R. Ratcliff, T. A. Gally, and R. L. Campbell, "Inverse wing design in transonic flow including viscous interaction," in Transonic Symposium: Theory, Application, and Experiment., NASA CP 3020, Vol. I, Part 2, 1989, pp. 497-520.

7 M. R. Hicks, M. E. Murman, and G. N. Vanderplaats, "An assessment of airfoil design by numerical optimization," NASA TM-3092, 1974.

8 G. A. Wrenn, "An indirect method for numerical optimization using the kreisselmeier-steinhauser function," NASA CR-4220, 1989.

9 G. B. Cosentino and T. L. Holst, "Numerical optimization design of advanced transonic wing configurations," NASA TM-85959, 1984.

10 K. D. Lee and S. Eyi, "Transonic airfoil design by constrained optimization," AIAA 9th Applied Aerodynamics Conference, September 1991, Baltimore, MD. AIAA 91-3287.

11 A. C. Taylor, G. W. Hou, and V. M. Korivi, "Sensitivity analysis, approximate analysis, and design optimization for internal and external viscous flows," AIAA Aircraft Design Systems and Operations Meeting, September 1991, Baltimore, MD. AIAA 91-3083.

12 A. Jameson, "Aerodynamic design via control theory," Journal of Scientific Computing, Vol. 3, 1988, pp. 233260.

13 A. Jameson, "Automatic design of transonic airfoils to reduce the shock induced pressure drag," in 31st Israel Annual Conference on Aviation and Aeronautics, February 21-22, 1990.

14 S. Ta'asan, "one shot methods for optimal control of distributed parameters systems i: Finite dimensional control," ICASE Report No. 91-2, 1991.

15 S. Ta'asan, G. Kuruvila, and M. D. Salas, "Aerodynamic design and optimization in one shot," AIAA 30th Aerospace Sciences Meeting \& Exhibit, January, 1992, Reno, NV. AIAA Paper 92-0025.

16 K. Karamcheti, Principles of Ideal-Fluid Aerodynamics. Robert E. Krieger Publishing Company, 1980.

17 C. Hirsch, Numerical Computation of Internal and External Flows. Vol. 1. John Wiley \& Sons, 1990.

18 A. Brandt, "Multilevel adaptive solutions to boundaryvalue problems," Mathematics of Computation, Vol. 31, 1977, pp. 333-390.

19 D. Althaus and F. X. Wortmann, Stuttgarter Profilkatalog I. Friedr. Vieweg \& Sohn, Braunschweig, West Germany, 1981, pp. 96-97. 